\title{
Theoretical basis for calculation of the quarries sides for collapse
}

\author{
Andrey Zhabko*, Natalya V olkomorova, and Natalya Zhabko \\ 1 U ral State M ining U niversity, 620144, Ekaterinburg, Russia
}

\begin{abstract}
The paper is devoted to the development of a mathematical apparatus for cal-culation of the quarries sides for collapse. The relevance of the paper lies in the fact that today there are no methods for calculation of the quarries and open-pits sides for collapse, however, the collapse of the sides or ledges in quarries is the most dangerous and unpredictable process. The main idea of the paper is to use, when calculating the collapse, not a strength criterion, but a criterion that describes the elastic limit of rocks. According to modern con-cepts, the formation of extremely stressed zones in the entire loaded region begins exactly at the elastic limit, in contrast to the ultimate strength, upon reaching which a single fracture surface is formed. Thus, the ultimate strength should be identified with landslide phenomena, and the elastic limit with the collapse of the rock mass. Methods of mathematical analysis and continuum mechanics method of ultimate stress state, are widely used in the paper.
\end{abstract}

\section{Introduction}

The most common and dangerous types of quarries sides deformation are landslides and collapses. Fundamental differences between these two types of deformations are different flow rates and places of localization. A landslide is a quite long-term process that allows to make a decision to strengthen the slope, and associated with the displacement of the landslide prism along a certain surface, which is commonly called the sliding surface. That is, the limiting condition is satisfied along a certain surface. As for the collapses, they proceed in an avalanche-like manner, which predetermines their danger. And, unlike landslides, collapses are caused by a loss of strength at each point of the collapsed mass. In other words, with a certain combination of the shape of the slope surface and the external load, some part of the adjacent rock mass can be in the ultimate stress state, which can lead to its collapse.

Historically, the science of the slopes stability has been developing in two directions, which G.L. Fisenko [1] called the method of ultimate equilibrium and the method of ultimate stress state. The method of ultimate equilibrium involves finding the most stressed sliding surfaces in the mass and comparisson of the shear and holding stresses along it and, in this regard, is a method of landslide phenomena preventing. The method of ultimate stress state implies the fulfillment of the differential equations of Navier equilibrium and

\footnotetext{
${ }^{*}$ Corresponding author: zhabkoav@ mail.ru
} 
the limit Coulomb condition at each point of the collapsing prism. This method was developed by the efforts of outstanding scientists K. Coulomb (1773) [2], V. Renkin (1857) [3], F. Ketter (1903) [4]. In a more or less final form, theoretically (analytically) is was designed by V.V. Sokolovsky (1939). In his fundamental work known as "Statics of granular medi-um" [5].

Judging by the publications, interest in this area of research has declined in recent years, with the exception of some works, for example [6]. However, the relevance of this issue is increasing due to the increasing depth of existing quarries. Paradoxically, but the current normative document on the stability of quarries and open pits [7] does not say anything about the calculation of slopes for collapse, and there are objective reasons for this.

In fig. 1, as an example, slopes calculated using the method of ultimate stress state (Renkin - Ketter - Sokolovsky) are given. The load size is combined with the bulk density of the slope.

Obviously, the implausibility of the calculation results that contradict any experimental and field data, despite the importance and rigor of the mathematical conclusions obtained by the above authors, obviously follows from the figures.

We will try to deal with the causes of this discrepancy. It is completely obvious that in the framework of continuum mechanics, the differential equations of equilibrium are flawless. In this regard, the reasons can only be found in the condition of ultimate equilibrium.
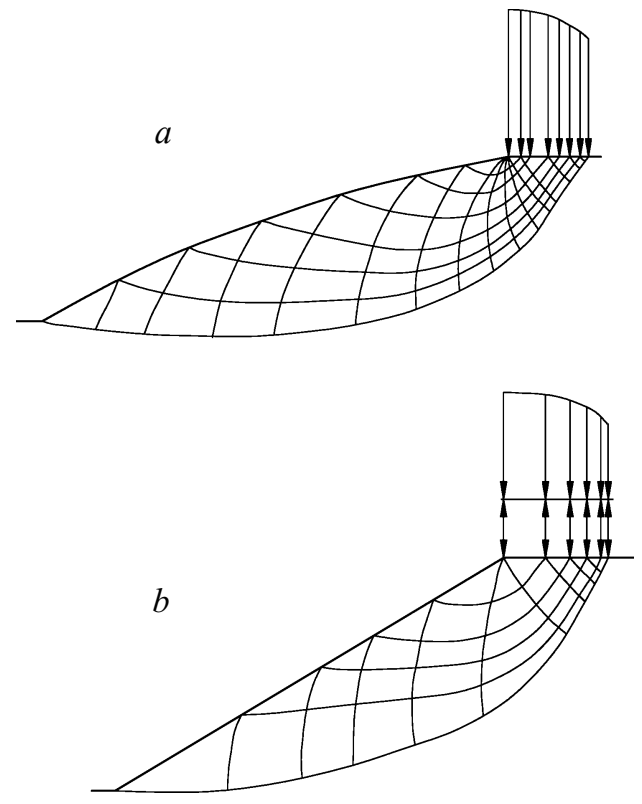

Fig. 1. Convex (a) and flat (b) slopes with a calculated weight load.

Firstly, at the ultimate strength, which is described by the Coulomb criterion, according to modern concepts, a general fault is formed (microshear sites are "excluded" from the deformation process). The shear sites begin to form already at the elastic limit and in the process of hardening. In the process of plastic hardening, complex processes of deformation of the translational-rotational nature occur, giving rise to the phenomenon of dilatancy, in which the material loses its continuity, expanding in size. Therefore, the continuum formulas in the hardening phase (and even more at the ultimate strength) do not reflect reality, as, for example, in the elastic phase. Moreover, it was experimentally proved [8] 
that the ultimate strength of rocks and artificial materials, with rare exceptions, are described by the Coulomb criterion. As a rule, the region of real strength of materials lies inside the Coulomb passport; which is a consequence of not taking into account dilatant changes during plastic deformation, and the elastic region, of course, is even smaller. That is, the association of elastic and strength limits is not legitimate! Unfortunately, almost everywhere in all geomechanical calculations, including mathematical modeling, the fulfillment of the Coulomb criterion is considered as the beginning of the exit of the material into the plastic phase (elastic limit).

Secondly, there is a reason to believe that the orientation of the critical shear sites depends on the level of the stress state, that is, there is a dependence on the stress deviator.

\section{Rock elasticity criterion}

In works $[8,9,10]$, on the basis of continuum representations, an analytical criterion was obtained, analyzed and experimentally verified that determines the elastic limit and ultimate strength of rocks, which, in the components of the main normal stresses, has the following form:

$$
\sigma_{3}=\sigma_{1}-2 C \sqrt{\left(1+\frac{\operatorname{tg} \varphi \sigma_{1}}{C}\right)\left(1+\frac{\operatorname{tg} \varphi k \sigma_{3}}{C}\right)},
$$

where $\sigma_{3}, \sigma_{1}$ - main stresses; $C$ - adhesion; $\varphi$ - angle of internal friction ( $f=\operatorname{tg} \varphi-$ coefficient of internal friction); $0 \leq k \leq 1$ - parameter that takes into account the dilatancy of the rocks and determines, for various particular values, the elastic limit, strength and plastic potential function.

It was experimentally confirmed [8] that all the rocks $0<k<1$ and artificial materials in the plastic hardening phase fall into the range of values. When $k=1$, criterion (1) describes the Coulomb criterion in the components of the principal stresses, and the angle of inclination of the critical site to the minimum principal stress is $\frac{\pi}{4}+\frac{\varphi}{2}$, this fully complies with the representations of the ultimate stress state method. When $k=0$, equation (1) has the following form:

$$
\sigma_{3}=\sigma_{1}-2 C \sqrt{1+\frac{\operatorname{tg} \varphi \sigma_{1}}{C}}=\sigma_{1}-2 C \operatorname{tg} \psi,
$$

where $\psi$ - angle of inclination of the shear area to the minimum principal stress (variable, in contrast to the existing concepts).

Criterion (2) for some rocks determines the elastic limit and for all rocks and materials, without exception, describes the lower limit of the onset of plastic deformation and ultimate strength [8]. The upper bound for the elasticity and strength limits is described by criterion (1) at $k=1$.

The use of elasticity criterion (2) as the limiting condition eliminates the abovementioned discrepancies and guarantees a certain margin of safety for quite brittle materials.

\section{Theory of slopes calculation for collapse}

Using the elasticity criterion (2), as well as the well-known dependences of continuum mechanics (3) (4), 


$$
\begin{gathered}
\sigma_{1,3}=\frac{\sigma_{x}+\sigma_{y}}{2} \pm \sqrt{\left(\frac{\sigma_{x}-\sigma_{y}}{2}\right)^{2}+\tau_{x y}^{2}} \\
\operatorname{tg} 2 \alpha=\frac{2 \tau_{x y}}{\sigma_{x}-\sigma_{y}}
\end{gathered}
$$

where $\sigma_{x}, \sigma_{y}, \tau_{x y}$ - components of the plane stress tensor; $\alpha$ - angle between the main sites and the axis $x$, we obtain the following equations:

$$
\begin{gathered}
\tau_{x y}=C \sin 2 \alpha \operatorname{tg} \psi . \\
\frac{\sigma_{x}-\sigma_{y}}{2}=C \cos 2 \alpha \operatorname{tg} \psi . \\
\frac{C}{\operatorname{tg} \varphi}\left(\operatorname{tg}^{2} \psi-1\right)-\sqrt{\left(\frac{\sigma_{x}-\sigma_{y}}{2}\right)^{2}+\tau_{x y}^{2}}=\frac{\sigma_{x}+\sigma_{y}}{2} .
\end{gathered}
$$

Thus, taking into account (5) - (7), we have the system of equations:

$$
\left\{\begin{array}{l}
\tau_{x y}=C \sin 2 \alpha \operatorname{tg} \psi \\
\frac{\sigma_{x}-\sigma_{y}}{2}=C \cos 2 \alpha \operatorname{tg} \psi \\
\frac{\sigma_{x}+\sigma_{y}}{2}=C\left(\frac{\operatorname{tg}^{2} \psi-1}{\operatorname{tg} \varphi}-\operatorname{tg} \psi\right)
\end{array}\right.
$$

Solving the system (8) with respect to the components of a plane stress field, we obtain:

$$
\left\{\begin{array}{l}
\tau_{x y}=C \sin 2 \alpha \operatorname{tg} \psi \\
\sigma_{x}=C\left(\frac{\operatorname{tg}^{2} \psi-1}{\operatorname{tg} \varphi}-(1-\cos 2 \alpha) \operatorname{tg} \psi\right) \\
\sigma_{y}=C\left(\frac{\operatorname{tg}^{2} \psi-1}{\operatorname{tg} \varphi}-(1+\cos 2 \alpha) \operatorname{tg} \psi\right)
\end{array}\right.
$$

We highlight that the angle of inclination of the critical site in the system of equations (9) $\psi$ is a variable. Let $\operatorname{tg} \psi=p(x, y)=p ; \cos 2 \alpha=q(x, y)=q$, then

$$
\left\{\begin{array}{l}
\tau_{x y}=C p \sqrt{1-q^{2}} \\
\sigma_{x}=C\left(\frac{p^{2}-1}{\operatorname{tg} \varphi}-(1-q) p\right) \\
\sigma_{y}=C\left(\frac{p^{2}-1}{\operatorname{tg} \varphi}-(1+q) p\right)
\end{array}\right.
$$


We place the functions (10) into the differential equations of plane equilibrium:

$$
\frac{\partial \sigma_{x}}{\partial x}+\frac{\partial \tau_{x y}}{\partial y}=0 ; \quad \frac{\partial \sigma_{y}}{\partial y}+\frac{\partial \tau_{x y}}{\partial x}=-\gamma,
$$

where $\gamma$ - volumetric weight of the rocks.

Thus, taking into account the adoption of the dimension of stress in adhesion units $(C=1)$, we will have the following system of equations:

$$
\left\{\begin{array}{l}
2 \operatorname{ctg} \varphi p \frac{\partial p}{\partial x}-\frac{\partial p}{\partial x}+p \frac{\partial q}{\partial x}+q \frac{\partial p}{\partial x}+\sqrt{1-q^{2}} \frac{\partial p}{\partial y}+p \frac{q}{\sqrt{1-q^{2}}} \frac{\partial q}{\partial y}=0 \\
2 \operatorname{ctg} \varphi p \frac{\partial p}{\partial y}-\frac{\partial p}{\partial y}-q \frac{\partial p}{\partial y}-p \frac{\partial q}{\partial y}+\sqrt{1-q^{2}} \frac{\partial p}{\partial x}+p \frac{q}{\sqrt{1-q^{2}}} \frac{\partial q}{\partial x}=-\gamma
\end{array}\right.
$$

By adding to the system (11) the equations for the total differentials of the sought functions,

$$
\left\{\begin{array}{l}
d p=\frac{\partial p}{\partial x} d x+\frac{\partial p}{\partial y} d y \\
d q=\frac{\partial q}{\partial x} d x+\frac{\partial q}{\partial y} d y
\end{array}\right.
$$

we obtain the basic system of equations of limit equilibrium:

$$
\left\{\begin{array}{l}
(2 \operatorname{ctg} \varphi p+q-1) \frac{\partial p}{\partial x}+p \frac{\partial q}{\partial x}+\sqrt{1-q^{2}} \frac{\partial p}{\partial y}+\frac{p q}{\sqrt{1-q^{2}}} \frac{\partial q}{\partial y}=0 \\
\sqrt{1-q^{2}} \frac{\partial p}{\partial x}+\frac{p q}{\sqrt{1-q^{2}}} \frac{\partial q}{\partial x}+(2 \operatorname{ctg} \varphi p-q-1) \frac{\partial p}{\partial y}-p \frac{\partial q}{\partial y}=-\gamma \\
d x \frac{\partial p}{\partial x}+0 \frac{\partial q}{\partial x}+d y \frac{\partial p}{\partial y}+0 \frac{\partial q}{\partial y}=d p \\
0 \frac{\partial p}{\partial x}+d x \frac{\partial q}{\partial x}+0 \frac{\partial p}{\partial y}+d y \frac{\partial q}{\partial y}=d q
\end{array}\right.
$$

To find the characteristics of the system (12), according to the well-known theory of solving hyperbolic systems in partial derivatives [11], it is necessary to solve the equation:

$$
\operatorname{det}\left(\begin{array}{cccc}
2 \operatorname{ctg} \varphi p+q-1 & p & \sqrt{1-q^{2}} & \frac{p q}{\sqrt{1-q^{2}}} \\
\sqrt{1-q^{2}} & \frac{p q}{\sqrt{1-q^{2}}} & (2 \operatorname{ctg} \varphi p-q-1) & -p \\
d x & 0 & d y & 0 \\
0 & d x & 0 & d y
\end{array}\right)=0 .
$$

The solution of equation (13) gives two real families of characteristics: 


$$
\frac{d y}{d x}=\frac{(f-2 p) \sqrt{1-q^{2}} \pm 2 \sqrt{f^{2} q^{2}\left(1-q^{2}\right)+p(p-f)}}{2 f q^{2}+(2 p-f) q-f} .
$$

Similarly, to find the ratio of functions on the system (12) characteristics, it is necessary to solve the following equation [11]:

$$
\operatorname{det}\left(\begin{array}{cccc}
2 \operatorname{ctg} \varphi p+q-1 & p & \sqrt{1-q^{2}} & 0 \\
\sqrt{1-q^{2}} & \frac{p q}{\sqrt{1-q^{2}}} & (2 \operatorname{ctg} \varphi p-q-1) & -\gamma \\
& 0 & d y & d p \\
0 & d x & 0 & d q
\end{array}\right)=0 .
$$

Thus, solving equation (15), the ratio on the characteristics has the following form:

$$
\begin{aligned}
& 4 \operatorname{ctg} \varphi d p=\frac{2 p-f(1+2 q)}{f-p} d q+\frac{d y}{d x} \frac{2 p q-f q+2 f q^{2}-f}{\sqrt{1-q^{2}}(f-p)} d q+ \\
& +\gamma d y \frac{2 p+f(q-1)}{p(f-p)}+\gamma d x \frac{f\left(q^{2}-1\right)}{\sqrt{1-q^{2}} p(f-p)} .
\end{aligned}
$$

In this manner, the joint solution of equations (14) and (16) will allow to determine the values of the functions $\operatorname{tg} \psi=p(x, y)=p ; \cos 2 \alpha=q(x, y)=q$ at each point of the extremely stressed region, as well as the boundary of this region and the external load necessary to fulfill the condition of limit equilibrium.

\section{Solution of boundary problems}

Let us consider as an example the problem of constructing the limiting form of a slope, in the case when a uniformly distributed load is applied on its upper horizontal platform (berm) . The rock characteristics taken into account are as follows: ; ; .

Fig. $2 \mathrm{~b}$ shows the extremely stressed region (prism of destruction), and Fig. 2 a shows a free concave slope according to V.V. Sokolovsky [5], for the same values of phys-ical and mechanical characteristics of rocks. It should be noted that the proposed solution has a number of important fundamental differences in comparison with the solution of V.V. Sokolovsky [5].

Firstly, the obtained area of possible outfalls and collapses has a more plausible form, which we often observe in real conditions, which cannot be said about the shape of the slope and the prism of destruction according to V.V. Sokolovsky (see Fig. 2 a). Secondly, in the solution of V.V. Sokolovsky [5] the slope is free, that is, without applied stresses. In the proposed solution, to fulfill the condition of ultimate equilibrium, slope loading is neces-sary. Its role in real conditions is played by rocks, for example, blasted rock mass. While excavation of the rock mass, that is, a decrease , a dump area will be formed in the massif, which may collapse when the excavator approaches the bottom. 


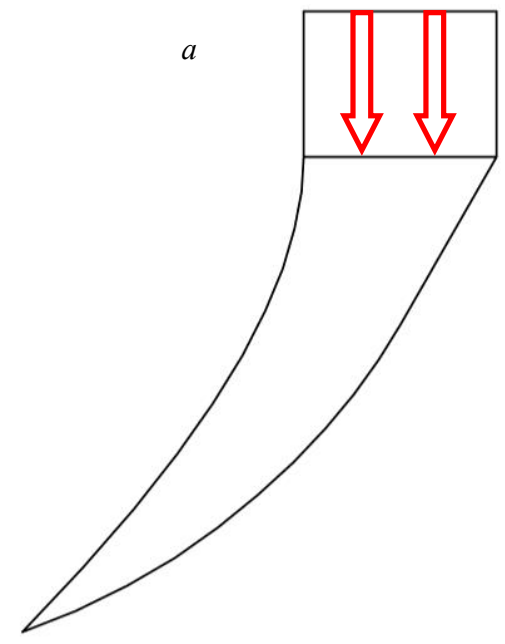

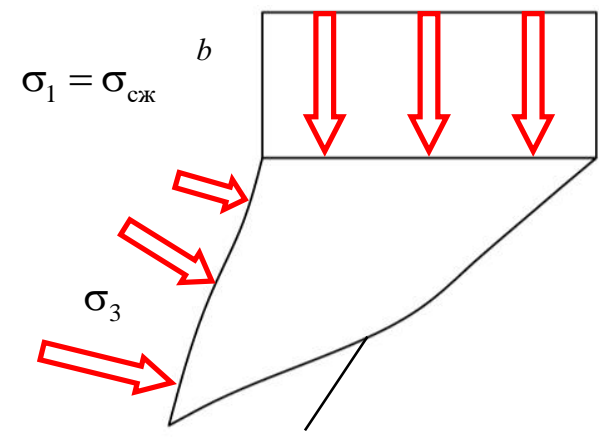

Not the sliding surface!!! This surface limits the region in which the rocks are in extreme stress state

Fig. 2. Extremely intense slope (prism) according to V.V. Sokolovsky [5] (a) and built using the proposed mathematical apparatus (b)

In addition, in contrast to the solution of V.V. Sokolovsky [5], where the characteristics of the system of equations coincide with the sliding surfaces, in the proposed solution, the characteristics are not sliding surfaces, but limit the region in which the condition of limit equilibrium is satisfied.

As noted above, issues of forecasting collapses and calculating slopes for collapses are not adequately reflected in Russian scientific and regulatory literature. The analysis of authoritative foreign scientific collections and monographs devoted to the issues of the slope stability [12 - 14] confirms this fact. Moreover, the guide open pits engineering [15], which, as indicated, contains comprehensive information on all design issues and developed by leading experts in the field of sustainability of the sides of open pits in Australia, the USA, Canada, South Africa, Chile, England, New Zealand, etc., also does not contain information on methods for slopes for collapse calculation. All this indicates the absence of a theory (mathematical apparatus) for calculating rock mass for collapse. In the framework of this work, a theory is proposed for calculating the stability of quarry sides for collapse.

\section{References}

1. G.L. Fisenko, Stability of the quarries and dumps sides, (1965)

2. C. Coulumb, Application des regles de maximis et minimis a quelques problemes de staticue relatifs a l'architecture, (1773)

3. W. Rancine, On the stability of loose earth, (1857)

4. F. Kötter, Die Bestimming des Druckes an gekrümmten Gleitflächen, eine Aufgabe aus der Lehre vom Erddruck, (1903)

5. V.V. Sokolovsky, Statics of a loose medium, (1990)

6. A.I. Chanyshev, G.M. Podyminogin, Fundamental and Applied Questions of Mining Sciences, 2, 291 (2015)

7. Rules for ensuring the stability of slopes in coal mines, (VNIMI, 1998)

8. A. V. Zhabko, USMU News, Theoretical and experimental aspects of plastic deformation and fracture of rocks, 1 (49), 68 (2018)

9. A. V. Zhabko, USMU News, Laws of plastic deformation and destruction of solids, 2 (46), 82 (2017) 
10. A. V. Zhabko, USMU News, Continuous concept of shear disintegration of solids, 2 (46), 111 (2019)

11. S.K. Godunov, Equations of Mathematical Physics, (1979)

12. Slope Stability Reference Guide for National Forests in the United States, Volume II. United States Department Agriculture, Engineering Staff, (Washington, DC, 1994)

13. Slope Stability, Department of the Army, U.S. Army Corps of Engineering, Washington, DC (2003)

14. L.W. Abramson, T.S. Lee, S. Sharma, G.M. Boyse, Slope Stability and stabilization methods, New York, (2002)

15. J. Read, P. Stacey, Guidelines for open pit slope design, (2009) 